\title{
Family Contact and Housing Stability in a National Multi-Site Cohort of Homeless Adults with Severe Mental Illness
}

\author{
Susan A. Pickett-Schenk · Judith A. Cook • Dennis D. Grey • \\ Sarah B. Butler
}

Received: 15 March 2007/Accepted: 22 March 2007/Published online: 1 June 2007

(C) Springer Science+Business Media, LLC 2007

\begin{abstract}
This study examined associations between family contact and housing stability among 4,778 homeless persons with mental illness who received intensive outreach and case management services through the national multi-site ACCESS project. Ordinary-least squares regression analysis found that, at 12-months poststudy entry, greater contact with relatives to whom participants feel close, more frequent telephone contact, and greater satisfaction with family relationships were associated with a greater number of nights in stable housing. Program and policy implications for strengthening family ties as a means to reduce homelessness among this population are discussed.
\end{abstract}

Keywords Family support · Homeless · Mentally ill

\section{Introduction}

Family support is frequently identified as a key ingredient in reducing the risk of homelessness among persons with mental illness (Cohen et al. 1997; Dixon et al. 1998; Pickett-Schenk 2002; Toro et al. 1999). Families are a major source of care for many adults with mental illness, providing both practical assistance and emotional support (Baronet 1999; Cook et al. 1999; Marsh and Johnson 1997; Ohaeri 2002). Little is known, however, about the role this support may play in strengthening homeless individuals' ability to maintain stable housing.

\footnotetext{
S. A. Pickett-Schenk $(\bowtie)$ J. A. Cook · D. D. Grey · S. B. Butler

Department of Psychiatry, University of Illinois at Chicago, 1601 W. Taylor Street, Chicago,

IL 60612, USA

e-mail: pickett@psych.uic.edu
} 


\section{Review of the Literature}

Findings related to family contact and support among homeless persons with mental illness are mixed. Compared to non-disabled homeless individuals, those with mental illness report less contact with their relatives, fewer supportive family relationships, and greater conflict with family members (Caton et al. 1994, 1995; Tessler et al. 1992; Toro et al. 1999). Other studies show that most homeless persons with mental illness are in contact with their families, feel that they can rely on family members for assistance, and have relatives to whom they feel close (Clarke et al. 1995; Dixon et al. 1998; Lam and Rosenheck 1999). This research also suggests that contextual characteristics, such as age, gender, race, and duration of homelessness influence family support, with younger individuals, women, African Americans, and those with shorter durations of homelessness reporting greater family contact and supportive relationships (Baré and Toro 1999; Caton et al. 1994, 1995; Lam and Rosenheck 1999; Wolch et al. 1993).

Few studies have examined the role of family contact and support in improving housing stability among homeless persons with mental illness. Wood et al. (1998) examined changes in family support among 316 homeless persons receiving case management services and access to Section 8 housing. At study entry, $32 \%$ of participants had seven or more days of contact (defined as in-person, phone, or mail contact) with a family member in the past 60 days. They had, on average, low levels of satisfaction with family relationships and low perceived availability of family members to provide instrumental and emotional support. At the 3-year follow-up, $51 \%$ reported seven or more days of contact with a family member in the past 60 days; and mean satisfaction and perceived availability of family support scores had increased significantly. High levels of contact were significantly associated with satisfaction with family relationships and perceived availability of practical and emotional assistance. Participants also experienced significant increases in housing stability, which was defined as residing independently in an apartment or in community housing for $80 \%$ or more of the follow-up period. Although improved housing stability was a significant predictor of increased family contact, the authors did not test the converse. That is, they did not examine whether changes in family support were associated with housing stability.

Calsyn and Winter (2002) used structural equation modeling to examine the causal relationships between natural support and housing stability among 3,930 homeless persons with mental illness who participated in the Access to Community Care and Effective Supports and Services (ACCESS) program, a federal research demonstration project, described in the Method section. Participant outcomes were assessed at study entry, and at 3-months and 12-months post-baseline. Natural support was defined as the amount of in-person contact with family members, friends, and co-workers; housing stability was assessed as the number of nights participants spent in an apartment or home in the 60 days prior to each assessment point. Participants' natural support and housing stability significantly increased over time. Natural support was positively associated with stable housing at 3-months and 12-months post-study entry, with greater contact resulting in a greater number of nights spent in stable housing in the 2 months prior to the follow-up period. 
However, the natural support measure combined contact with kin and non-kin, and thus the true effect of family contact on housing stability could not be determined from this study's results.

The present study extends this prior research by examining associations between family contact, support, and satisfaction with family relationships and housing stability among ACCESS program participants. We predicted that: (1) a higher frequency of face-to-face contact with close family members would be associated with a greater number of days in stable housing; (2) a higher frequency of telephone contact with relatives would be associated with a greater number of days in stable housing; and (3) higher satisfaction with level of family contact would be associated with a greater number of days in stable housing, and that these relationships would be significant in the presence of participant contextual characteristics.

\section{Method}

\section{ACCESS Demonstration Program}

Conducted from 1994 to 1998, ACCESS was the largest and most rigorous study of the effect of service systems integration on the use of services, health status, and quality of life of homeless persons with mental illness (Randolph et al. 1997, 2002). ACCESS provided intensive outreach and case management services to over 7,000 homeless persons with mental illness at 18 sites across the country. All sites used an interdisciplinary team of staff (i.e., social workers, psychiatrists, substance abuse counselors) to deliver a wide array of community-based services to participants. These services included: housing assistance, medical and dental care, medication evaluation and management, alcohol and drug treatment, counseling, employment assistance, and public entitlement procurement assistance (Johnsen et al. 1999). One year post-program enrollment, improved outcomes for ACCESS participants included a greater number of days in independent housing; fewer alcohol and drug problems; decreased psychiatric symptoms; improved quality of life; and greater service use (Rosenheck et al. 1998, 2002).

\section{Sample}

A total of 7,229 individuals enrolled in the ACCESS study (Rosenheck et al. 2002). The sample used in this analysis consists of 4,778 ACCESS participants who had complete baseline, 3 months, and 12-month follow-up data. As shown in Table 1, of these 4,778 participants, $61 \%$ were men and $39 \%$ women. Just over half $(51 \%)$ were African American; 41\% were Caucasian; 4\%, Hispanic/Latino; 2\%, Native American; 2\%, other (i.e., mixed race); and less than $1 \%$ Asian/Pacific Islander. Over a third $(38 \%)$ had not graduated from high school, another third $(36 \%)$ had a high school diploma or General Equivalency Degree (GED), while 27\% had some college education or a college degree. Only a small minority $(5 \%)$ reported that they were married. Participants' average age at baseline was 38 years (ranging from 
Table 1 Demographic characteristics of ACCESS participants at baseline $(N=4,778)$

\begin{tabular}{|c|c|c|}
\hline Variables & $N$ & $\%$ \\
\hline \multicolumn{3}{|l|}{ Gender } \\
\hline Male & 2,901 & 60.7 \\
\hline Female & 1,877 & 39.3 \\
\hline \multicolumn{3}{|l|}{ Ethnicity } \\
\hline African American & 2,415 & 50.6 \\
\hline Asian, Pacific Islander & 30 & .6 \\
\hline Caucasian & 1,950 & 40.9 \\
\hline Hispanic & 210 & 4.4 \\
\hline Native American & 83 & 1.7 \\
\hline Other & 85 & 1.8 \\
\hline \multicolumn{3}{|l|}{ Education } \\
\hline Less than high school & 1,799 & 37.7 \\
\hline High school graduate or higher & 2,979 & 62.3 \\
\hline \multicolumn{3}{|l|}{ Diagnosis } \\
\hline Schizophrenia & 2,601 & 36.4 \\
\hline Other psychotic disorder & 2,268 & 31.7 \\
\hline Major depression & 3,493 & 48.8 \\
\hline Bipolar disorder & 1,464 & 20.5 \\
\hline Personality disorder & 1,572 & 22.0 \\
\hline PTSD & 1,070 & 15.0 \\
\hline Anxiety disorder & 1,298 & 18.2 \\
\hline Adjustment disorder & 905 & 12.7 \\
\hline Alcohol dependency & 3,065 & 42.9 \\
\hline Drug dependency & 2,752 & 38.5 \\
\hline Other & 1,513 & 21.2 \\
\hline Mean $( \pm \mathrm{SD})$ [range] age in years & $38.3(9.5)$ & [18-84] \\
\hline Mean $( \pm \mathrm{SD})$ [range] lifetime duration of homelessness in years & $6.9(8.4)$ & {$[<1-62]$} \\
\hline Mean $( \pm$ SD) number of prior hospitalizations & $4.8(12.3)$ & \\
\hline
\end{tabular}

18 years to 84 years). On average, they had been homeless for a total of 7 years lifetime (ranging from less than 1 year to a high of 62 years).

Three months post-enrollment, case managers were asked to select diagnoses for their clients from a list of 14 DSM-IV axis I and axis II diagnoses developed by the study investigator (Fisher and Breakey 1991). Nearly half (49\%) of the clients were thought by their case managers to have major depression; $43 \%$ were thought to be alcohol dependent; $39 \%$, to drug dependent; $36 \%$ to have schizophrenia; $32 \%$ to have another psychotic disorder (e.g., schizoaffective disorder, delusional disorder, psychotic disorder not otherwise specified (schizoaffective disorder, or delusional disorder); $22 \%$ to have a personality disorder; and $21 \%$ to have bipolar disorder. More than half of the ACCESS clients (55\%) had a single diagnosis; $26 \%$ had two diagnoses; and 19\% had three diagnoses. Almost three-quarters (73\%) had been 
hospitalized for mental health problems at some point in their lifetimes before baseline, with an average of five prior hospitalizations.

\section{Procedures}

To enroll in the study, individuals were required to meet study inclusion criteria for mental illness and homelessness. Eligibility criteria were assessed by ACCESS research staff during the outreach and engagement process. To determine presence of psychiatric illness, a 30-item screening instrument (Shern et al. 1994) was used, which measured depressive, psychotic, and manic symptoms. Homelessness was assessed as having spent at least seven of the past 14 days prior to initial contact on the streets, in a shelter, in a car, or in an abandoned building (Rosenheck and Lam 1997). Those who met eligibility criteria were invited to join the study and receive ACCESS services.

\section{Measures}

The ACCESS protocol included three in-person interviews administered at the time of study enrollment and then 3 months and 12 months afterwards. Interviews lasted approximately $1-1 \frac{1}{2} \mathrm{~h}$, and were conducted by researchers who were not part of the ACCESS clinical staff. Participants received $\$ 15$ for each interview and were afforded human subjects protection and confidentiality safeguards in accordance with Institutional Review Board requirements. The ACCESS protocol assessed several areas of participants' lives including their history of homelessness, current housing status, psychiatric history and treatment, service utilization, and sociodemographic features. In this study, variables measuring history of homelessness, current residential status, level and nature of family contact, clinical history, and background demographic characteristics were used.

\section{Housing Stability}

The dependent variable of housing stability was operationalized as the number of days out of the past 60 days living in one's own or another's house, apartment, or rooming house (Rosenheck et al. 1998).

\section{Family Contact}

Three single-item family contact measures served as independent variables and included in-person contact, satisfaction with contact, and telephone contact. Inperson contact was assessed as the frequency of face-to-face contact the participant had with people he/she felt close to who were members of his/her family of origin (parents, grandparents, and siblings) and/or family of procreation (children and spouse/significant other). Responses were coded on a scale ranging from never (0) to (5) daily (Leda and Rosenheck 1992, 1995) and recoded for this study into three categories as daily, weekly, or less than weekly. An item assessing satisfaction with family contact was adapted from the Lehman Quality of Life Instrument (Lehman 
1988) and asked participants to rate their level of satisfaction with the amount of contact they had with their family, coded on a scale ranging from delighted (7) to terrible (1). Telephone contact was measured as the frequency of telephone contact with relatives using an item that was coded as daily (4), weekly (3), monthly (2), less than monthly but at least once per year (1), and none (0).

\section{Control Variables}

Gender, race, age, education, homelessness history, psychiatric history, and family co-residence served as study control variables. Gender was coded as 1 for males and 0 for females. Race was coded as 1 if participants were racial minorities and 0 if they were Caucasians. Age was measured in years. Education level was coded as 1 if participants had graduated from high school or received their GED, and 0 if they had not. Homelessness history was assessed as the number of years that participants had been homeless at the time of study entry. Psychiatric history was assessed as total number of lifetime inpatient admissions participants reported at enrollment. Because of potential confounding with the study's family contact variables, family co-residence was controlled for in the analysis and measured as a dichotomous variable coded 1 if participants lived with relatives and 0 if not.

\section{Analysis}

The analysis involved inspection of frequency distributions and descriptive statistics for all study variables. Next, ordinary least squares (OLS) regression analysis was used to examine the association between housing stability at 12-month follow-up and family contact variables, while controlling for other multivariate predictors. Significant associations between housing status and family contact were then examined using one-way repeated-measures analysis of variance to test for differences between those in stable housing versus not by nature and frequency of family contact at the three study time points.

\section{Results}

\section{Housing Stability}

At 12-month follow-up, respondents reported spending an average of 41.7 days out of the past 60 days living in their own or another person's house, apartment, or room. This ranged from 0 days to 60 days, with a median of 60 , a mode of 60 , and standard deviation of 25.5. This was a significant increase $(p<.001)$ over this same figure at 3 months post-baseline, at which point respondents reported an average of 30.6 days living independently, a median of 30 , mode of 60 , and standard deviation of 26.6. At 12-month follow-up, a fifth of the respondents $(20 \%, n=966)$ reported residing with one or more family members. 


\section{Family Contact}

Individuals were asked to report the frequency of face-to-face contact with any relative they felt close to by category of family member (e.g., parent, sibling, offspring, spouse/partner). There was little variation in face-to-face contact by family relationship: $33 \%$ of respondents reported in-person contact with spouses/ significant others; $29 \%$ reported face-to-face contact with their children; $27 \%$ had face-to-face contact with their parents; and $21 \%$ had in-person contact with siblings. Thus, the highest frequency of contact was used to characterize each respondent, so that an individual reporting daily contact with a spouse and monthly contact with offspring was classified as having daily contact with at least one close relative. At 12-month follow-up, $28 \%$ of respondents $(n=1338)$ reported daily face-to-face contact with one or more close relatives, $15 \%(n=706)$ reported weekly face-toface contact with close relatives, $11 \%(n=524)$ reported monthly contact, $5 \%$ $(n=269)$ less than monthly but at least several times per year, 6\% $(n=275)$ hardly ever, and 35\% ( $n=1,662)$ reported never having face-to-face contact with a close relative. When asked to rate their degree of satisfaction with the amount of family contact along a continuum, $7 \%(n=340)$ rated themselves as delighted, $14 \%$ $(n=663)$ pleased, 19\% $(n=865)$ mostly satisfied, $19 \%(n=900)$ mixed, $12 \%$ $(n=576)$ mostly dissatisfied, $16 \%(n=718)$ unhappy, and 13\% $(n=584)$ terrible. Regarding telephone contact with any family members (regardless of the closeness of their relationships), $12 \%(n=593)$ reported daily phone contact, $25 \%(n=1,182)$ weekly contact, $19 \%(n=880)$ monthly contact, $16 \%(n=762)$ less than monthly but at least once per year, and 28\% $(n=1323)$ reported no phone contact with a relative in the past year.

\section{Multivariate Predictors of Housing Stability}

Next, we examined associations between residential stability at 12-month follow-up and family contact variables, while controlling for a series of demographic variables, clinical factors, history of homelessness, and co-residence with family members. As shown in Table 2, OLS regression analysis found that those who reported that they saw close family members daily had a significantly greater number of days in stable housing than those who saw close relatives monthly or less often. There was also a trend $(p=.051)$ in which those who saw close family weekly were more likely to have successful housing outcomes than those who saw their close relatives monthly or less often. Those who reported higher levels of satisfaction with the amount of contact they had with family members had significantly greater number of days in stable housing than those who were dissatisfied with their level of contact with close relatives. In addition, the greater the frequency of telephone contact with relatives, the greater the number of days in stable housing experienced by respondents at 12-month follow-up. These associations persisted even controlling for whether the respondent lived with family members, their history of homelessness, number of psychiatric hospitalizations, and demographic variables such as gender, race, age, and education. In a separate analysis (not shown) we controlled for the respondent's diagnosis nominally and 
Table 2 Multivariate analysis (ordinary least squares) of associations between housing stability ${ }^{\mathrm{a}}$ and family contact among homeless individuals at 12-month follow-up

\begin{tabular}{lccc}
\hline Variables & $B$ & Beta & $p$-Value \\
\hline Male & -5.828 & -.112 & .000 \\
Minority status & -4.453 & -.88 & .000 \\
Age & 1.197 & .044 & .003 \\
Completed high school & 1.033 & .020 & .174 \\
Years homeless (lifetime) at baseline & -.050 & -.016 & .281 \\
Times in psychiatric hospital at baseline & -.024 & -.008 & .587 \\
Daily face-to-face contact w close relative & 7.948 & .142 & .000 \\
Weekly face-to-face contact w close relative & 2.148 & .030 & .051 \\
Less than weekly face-to-face contact & Contrast & - & - \\
Satisfaction with amount of family contact & .658 & .047 & .002 \\
Frequency of telephone contact w family & 1.029 & .057 & .001 \\
Family member lives with subject & 5.850 & .084 & .000 \\
Constant & 34.094 & & \\
\hline$R^{2}=.089$, & & &
\end{tabular}

$R^{2}=.089, \mathrm{df}=11,4493, p<.001$

a Number of days out of the past 60 days living in one's own or another's house, apartment, or rooming house

found that it was not a significant addition to the model nor did it influence the significance of the three family variables.

\section{Changes in Family Contact over Time}

Although the multivariate analysis identified significant associations between positive housing outcomes and family contact, we wanted to determine whether level of contact changed over time in ways that were different for those in stable versus unstable housing. To do this, we divided the sample into those who had been living in their own or another person's house, apartment or rooming house for 60 out of the past 60 days versus those who had not done so. We used one-way repeatedmeasures ANOVA to test for differences between the two groups in changes regarding frequency, type, and satisfaction with family contact over time. Since participants entered the study with periods of recent homelessness, significant increases in family contact for those in stable housing versus not adds weight to the explanatory link between family contact and housing stability. As shown in Table 3, among those in stable housing, the proportion reporting daily contact with a close relative rose from $28 \%$ at baseline to $31 \%$ at 3 months and to $40 \%$ at 12 months. Concurrently, among the unsuccessfully housed, the proportions were $15 \%, 18 \%$, and $17 \%$ respectively. The ANOVA was significant, $F(1,4752)=111.18, p<.001$, indicating differential increases over time for the one group but not the other. Regarding satisfaction with family contact, among those in stable housing, 33\% reported themselves as satisfied with their level of contact at baseline, rising to $41 \%$ at 3 months, and $48 \%$ at 12 months. Among those in unstable housing, the 
Table 3 Change over time in family contact by housing stability ${ }^{\mathrm{a}}$ among homeless individuals with mental illness $(N=4,778)$

\begin{tabular}{|c|c|c|c|}
\hline Variables & Housing stability $\%$ or mean & Housing instability $\%$ or mean & ANOVA \\
\hline \multicolumn{4}{|c|}{ Sees close relative daily } \\
\hline Baseline & 28 & 15 & $F=111.18$ \\
\hline 3 months & 31 & 18 & $\mathrm{df}=1,4752$ \\
\hline 12 months & 40 & 17 & $p<.001$ \\
\hline \multicolumn{4}{|c|}{ Satisfied with amount of family contact } \\
\hline Baseline & 33 & 33 & $F=26.58$ \\
\hline 3 months & 41 & 40 & $\mathrm{df}=1,4609$ \\
\hline 12 months & 48 & 41 & $p<.001$ \\
\hline \multicolumn{4}{|c|}{ Frequency ${ }^{\mathrm{b}}$ of telephone contact with family } \\
\hline Baseline & 2.8 & 2.5 & $F=33.11$ \\
\hline 3 months & 2.8 & 2.6 & $\mathrm{df}=1,4729$ \\
\hline 12 months & 3.0 & 2.6 & $p<.001$ \\
\hline
\end{tabular}

a Stable housing $=$ resided in own/other's home, apartment, room for 60 out of past 60 days

b Frequency ranges from $5=$ daily, $4=$ weekly, $3=$ monthly, $2=$ less than monthly but at least once, $1=$ not at all

percentages satisfied with family contact were $33 \%, 40 \%$, and $41 \%$ respectively. The ANOVA here was significant, $F(1,4609)=26.58, p<.001$, as well, indicating greater increases in satisfaction with family contact over time for the stably housed group compared to those in unstable housing. Finally, we examined frequency of telephone contact with family, and found that it remained level between baseline and 3 months with an average score of 2.8, rising to an average score 3.0 at 12-month follow-up. Among those in unstable housing, telephone contact averaged 2.5 at baseline, rising to 2.6 at 3 months and remaining at 2.6 at 12 months, below the level of monthly contact. The ANOVA here was significant, $F(1,4729)=33.11$, $p<.001$, indicating that telephone contact increased among those in stable housing to a greater extent than among those in unstable housing. Thus, for all family contact variables, improvement over time was significantly greater for those in stable housing than for those in unstable housing.

\section{Discussion}

Study findings indicate that there are strong associations between housing stability and the frequency, type, and level of satisfaction with family contact among homeless individuals with severe mental illness. This was the case, regardless of the individual's demographic characteristics, history of homelessness, illness chronicity, or whether the individual resided with family members. In addition, we found significant increases over time in frequency of family contact and satisfaction with contact among those in stable housing compared to those who did not experience residential stability. Whereas we cannot make causal assertions, it may be that 
increased family contact, support, and satisfaction involve enhanced levels of family caregiving that allow homeless individuals to establish and maintain stable housing.

Results of this study suggest the importance of family reunification and support services for homeless persons with mental illness who have lost touch with or are estranged from their relatives and who desire such assistance. Given strong associations between family contact and housing stability, relatives can play a major role in supporting attempts to live independently in the community. For example, Dixon et al. (1998) found that the addition of a family outreach worker to an assertive community treatment (ACT) team was significantly associated with increased satisfaction with family relationships and stable housing. At one of the ACCESS sites in Illinois, a part-time family support worker (FSW) was hired in the latter years of the project, and worked with participants and family members to reestablish contact and repair estranged relationships (Pickett-Schenk 2002). The FSW helped negotiate discussions between participants and their family members about when and where contact would occur, and expectations for care, such as whether participants could be included in family gatherings or receive help with clothing. The FSW also educated families about the causes and treatment of mental illness, and how to cope better with the problems they and their ill relatives experienced. In addition to helping families understand better the experiences of their ill relatives, such services also may help homeless persons with mental illness deal more effectively with family members who are unsupportive or unhelpful, such as those who abuse substances or are prone to violence. Given these potential positive outcomes, the inclusion of a FSW on treatment teams that work with homeless persons with mental illness is warranted.

There are several limitations to this study. With the exception of the site in Illinois, we do not know the extent to which (1) ACCESS staff worked with participants to reunite them with their families, if at all; and (2) ACCESS participants requested assistance with strengthening family relationships. The "prescribed" service mix at ACCESS sites included intensive outreach and an array of case management services designed to stabilize symptoms, prevent relapse, and improve community functioning (Johnsen et al. 1999). It is possible that sites included services such as those described above, but that these family servicesincluding those provided by the FSW in Illinois-were not directly assessed. Similarly, service needs and use measures in the ACCESS interview protocol did not include items assessing need for and use of family reunification services. Thus, although it is possible that some ACCESS participants may have requested help to strengthen family relationships, no data were available to allow us to test for the effect of receipt of these services on housing stability. Additionally, the protocol did not assess whether ACCESS participants received specific types of assistance from family members, and thus we cannot determine if changes in contact and the resulting effect on housing stability are due to increased receipt of familial instrumental versus emotional support. Over time, participants may have received increased financial assistance from families that helped them pay rent or buy necessities, as well as emotional support that promoted independent housing. 


\section{Policy Implications}

The report of the New Freedom Commission on Mental Health (2003) called for family-centered and family-driven care, in which relatives of individuals with mental illness receive the assistance they need to provide support to their ill relatives. This report suggested that family respite services would enhance the ability of family members to support their relative's recovery. Such family assistance is community-based, frequently provided in the home, and designed to alleviate the pressures of ongoing care. These services, provided in conjunction with education about mental illness, may help prevent homelessness as well as promote return to housing stability. Family members often report high levels of physical and psychological distress, financial burden, and strained relationships as a result of caring for adult relatives with mental illness. Programs that educate families about the clinical etiology and treatment of mental illness, help them access services, and promote self-care have been found to strengthen family members' coping ability (Dixon and Lehman 1995; Pickett-Schenk et al. 2006). Family members who are better equipped with practical knowledge and emotional support may be able to help their relative avoid homelessness altogether, serving a primary prevention role. Given evidence that family support is associated with greater ease in obtaining formal services by homeless individuals (Acosta and Toro 2000), such efforts also could enhance the likelihood that families will support homeless individuals' efforts to obtain needed services such as employment, education, and treatment for substance abuse and mental health problems. This, in turn, has the potential to increase the latter's overall functioning and community integration, and to reduce their dependence on caregiving provided by relatives. Thus, as supported by study findings, policies promoting family reunification and relationship enhancement services are needed to fully support the recovery of homeless persons with mental illness.

Acknowledgments This research was funded by cooperative agreement UD1SM1368 with the Center for Mental Health Services (CMHS), Substance Abuse and Mental Health Services Administration (SAMHSA); and by cooperative agreement H-133B-000700 with the National Institute on Disability and Rehabilitation Research, U.S. Department of Education, and CMHS, SAMHSA. Its contents do not reflect the official policy or position of any federal agency. The authors thank Robert Rosenheck, M.D., Frances Randoph, Dr. P.H., the ACCESS National Evaluation Team, the ACCESS local evaluators for their invaluable assistance with this project.

\section{References}

Acosta, O., \& Toro, P. (2000). Let's ask the homeless people themselves: A needs assessment based on a probability sample of adults. American Journal of Community Psychology, 28, 343-366.

Baré, D. S., \& Toro, P. A. (1999). Developing measures to assess social support among homeless and poor people. Journal of Community Psychology, 27(2), 137-156.

Baronet, A. M. (1999). Factors associated with caregiver burden in mental illness: A critical review of the research literature. Clinical Psychology Review, 19, 819-841.

Calsyn, R. J., \& Winter, J. P. (2002). Social support, psychiatric symptoms, and housing: A casual analysis. Journal of Community Psychology, 30(3), 247-259. 
Caton, L. M., Shrout, P. E., Dominguez, B., Eagle, P. F., Opler, L., \& Cournos, F. (1995). Risk factors for homelessness among women with schizophrenia. American Journal of Public Health, 85(8), 11531156.

Caton, L. M., Shrout, P. E., Eagle, P. F., Opler, L., Felix, A., \& Dominguez, B. (1994). Risk factors for homelessness among schizophrenic men: A case-control study. American Journal of Public Health, 84(2), 265-270.

Clarke, P. N., Williams, C. A., Percy, M. A., \& Kim, Y. S. (1995). Health and life problems of homeless men and women in the southeast. Journal of Community Health Nursing, 12(2), 101-110.

Cohen, C. I., Ramirez, M., Teresi, J., Gallagher, M., \& Sokolovsky, J. (1997). Predictors of becoming redomiciled among older homeless women. The Gerontologist, 37(1), 67-74.

Cook, J. A., Heller, T., \& Pickett-Schenk, S. A. (1999). The effect of support group participation on caregiver burden among parents of adult offspring with severe mental illness. Family Relations, 4(4), 405-410.

Dixon, L., \& Lehman, A. (1995). Family interventions in schizophrenia. Schizophrenia Bulletin, 21, 631-643.

Dixon, L., Stewart, B., Krauss, N., Robbins, J., Hackman, A., \& Lehman, A. (1998). The participation of families of homeless persons with severe mental illness in an outreach intervention. Community Mental Health Journal, 34(3), 251-259.

Fischer, P. J., \& Breakey, W. R. (1991). The epidemiology of alcohol, drug, and mental disorders among homeless persons. American Psychologist, 46, 1115-1128.

Johnsen, M., Samberg, L., Calsyn, R., Blasinksy, M., Landow, W., \& Goldman, H. (1999). Case management models for persons who are homeless and mentally ill: The ACCESS demonstration project. Community Mental Health Journal, 35(4), 325-345.

Lam, J. A., \& Rosenheck, R. (1999). Social support and service use among homeless persons with serious mental illness. International Journal of Social Psychiatry, 45(1), 13-28. .

Leda, C., \& Rosenheck, R. (1992). Mental health status and community adjustment after treatment in a residential treatment program for homeless veterans. American Journal of Psychiatry, 149, 12191224.

Leda, C., \& Rosenheck, R. (1995). Race in the treatment of homeless mentally ill veterans. Journal of Nervous and Mental Disease, 183, 529-537.

Lehman, A. F. (1988). A quality of life interview for the chronically mentally ill. Evaluation and Program Planning, 11, 51-62.

Marsh, D. T., \& Johnson, D. L. (1997). The family experience of mental illness: Implications for intervention. Professional Psychology: Research \& Practice, 28(3), 229-237.

New Freedom Commission on Mental Health. (2003). Achieving the promise: Transforming mental health care in America. Final Report [DHHS Pub. No. SMA-03-3832]. Rockville, MD: New Freedom Commission on Mental Health.

Ohaeri, J. (2002). The burden of caregiving in families with mental illness: A review of 2002. Current Opinions in Psychiatry, 16, 457-465.

Pickett-Schenk, S. (2002). Families of homeless persons with mental illness. The Journal of NAMI California, 11(2), 77-78.

Pickett-Schenk, S., Cook, J. A., Steigman, P., Lippincott, R., Bennett, C., \& Grey, D. (2006). Psychological well-being and relationship outcomes in a randomized study of family-led education. Archives of General Psychiatry, 63, 1043-1050.

Randolph, F., Blasinksy, M., Leginski, W., Parker, L. B., \& Goldman, H. H. (1997). Creating integrated service systems for homeless persons with mental illness: The ACCESS Program. Psychiatric Services, 48, 369-373.

Randolph, F., Blasinksy, M., Morrissey, J. P., Rosenheck, R. A., Cocozza, J., Goldman, H. H., et al. (2002). Overview of the ACCESS program. Psychiatric Services, 53(8), 945-948.

Rosenheck, R., \& Lam, J. A. (1997). Homeless mentally ill clients' and providers' perception of service needs and clients' use of services. Psychiatric Services, 48(3), 381-386.

Rosenheck, R. A., Lam, J., Morrissey, J. P., Calloway, M.O., Stolar, M., Randolph, F., et al. (2002). Service systems integration and outcomes for mentally ill homeless persons in the ACCESS Program. Psychiatric Services, 53(8), 967-969.

Rosenheck, R., Morrissey, J., Lam, J., Calloway, M., Johnsen, M., Goldman, H., et al. (1998). Service system integration, access to services, and housing outcomes in a program for homeless persons with severe mental illness. American Journal of Public Health, 88(11), 1610-1615. 
Shern, D. L., Lovell, A. M., Tsemberis, S., Anthony, W., Lacomb, C. A., Richmond, L., et al. (1994). The New York City outreach project: Serving a hard-to-reach population. In making a difference: Interim status report of the McKinney demonstration program for homeless adults with serious mental illness. Rockville, MD: Center for Mental Health Services.

Tessler, R. C., Gamache, G. M., Rossi, P. H., Lehman, A. F., \& Goldman, H. H. (1992). The kindred bonds of mentally ill homeless persons. New England Journal of Public Policy, 8(1), 265-280.

Toro, P. A., Goldstein, M. S., Rowland, L. L., Bellavia, C. W., Wolfe, S. M., Thomas, D. M., et al. (1999). Severe mental illness among homeless adults and its association with longitudinal outcomes. Behavior Therapy, 30, 431-452.

Wolch, J. R., Rahimian, A., \& Koegel, P. (1993). Daily and periodic mobility patterns of the urban homeless. The Professional Geographer, 45(2), 159-169.

Wood, P. A., Hurlburt, M. S., Hough, R. L., \& Hofstetter, C. R. (1998). Longitudinal assessment of family support among homeless mentally ill participants in a supported housing program. Journal of Community Psychology, 26(4), 327-344. 